\title{
Dietary Shifts in Nepal and its possible impacts on overweight and obesity
}

\author{
Y. P. Subedi, D. Marais and D. Newlands \\ College of Life Sciences and Medicine, University of Aberdeen, Scotland, United Kingdom
}

The nutrition transition involves economic, demographic, dietary and epidemiological shifts, which are each affected by one another. ${ }^{(1)}$ Patterns and trends of Nepalese transitions are unknown. This study aimed to describe the economic, dietary and obesity shifts in Nepal by identifying economic time-series trends from government databases; dietary trends from food balance sheets and obesity shifts from family health and demographic health surveys. Trends were calculated at five yearly increments from available data from 1970 to 2010 .

The average proportion of energy from fat has significantly increased from 13\% in 1970 to $17 \%$ in 2010 ( $p<0.05$ ) (Figure 1). Plant fat and sugar are the main contributors to the increased energy intake trends over the 40 years, followed by meat, fish, milk and eggs. Data shows that overweight and obesity rates have increased from $21 \%$ of women to $27 \%$ in the urban population.
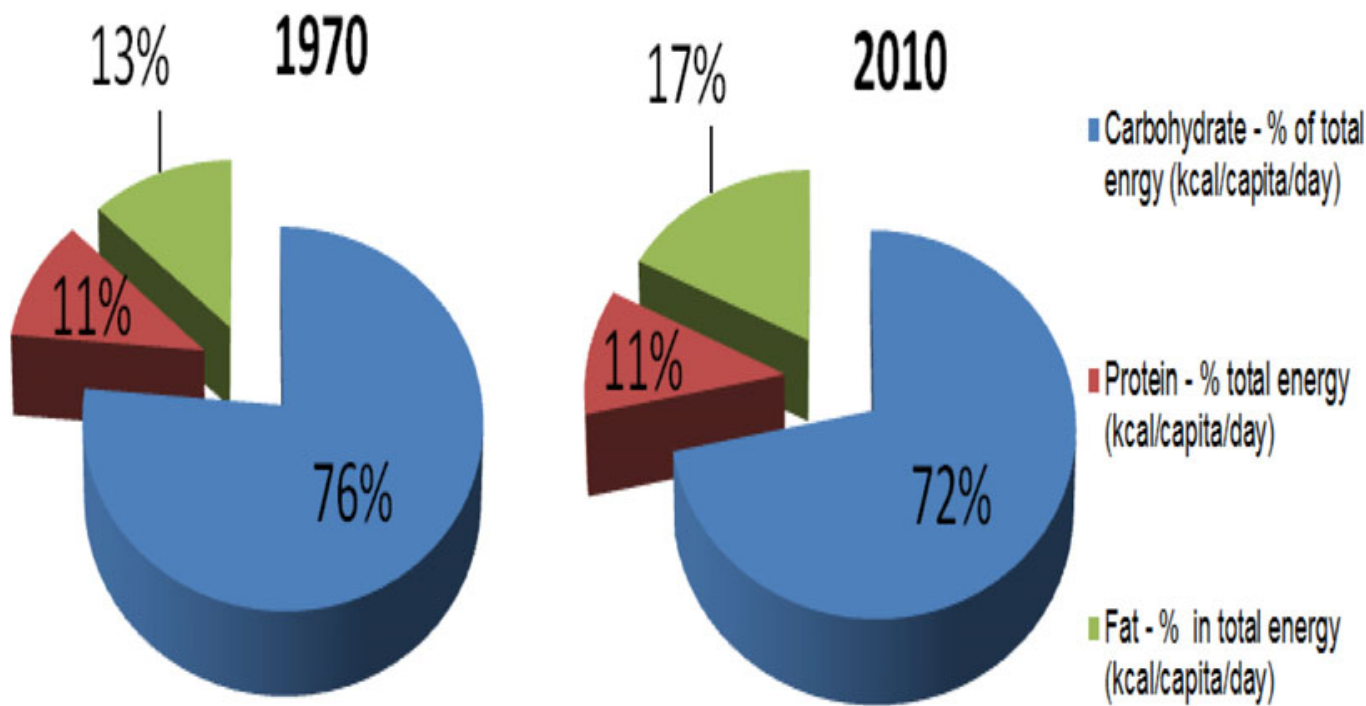

Fig. 1. Dietary shifts of macronutrient energy distribution from 1970 to 2010.

Nepalese dietary patterns have changed over the past forty years, especially with increased energy from plant fat, sugar and animal products coinciding with increased levels of obesity and overweight, especially in urban areas.

1. Popkin BM (2011) Contemporary nutritional transition: determinants of diet and its impacts on body composition, proceedings of the Nutrition Society 70, $1-82$. 